\title{
PEDRO FUNARI Y LA TRANSMISIÓN DEL CONOCIMIENTO
}

Margarita Díaz-Andreu ${ }^{1}$

Siempre he admirado a Pedro, al Prof. Funari, por su capacidad de trabajo y su entusiasmo, y por su habilidad de estar siempre rodeado de una cohorte de alumnos inteligentes, bien formados y bien disciplinados. Para los que venimos del Viejo Mundo, encontrarnos con tal nivel de calidad es siempre reconfortante. $\mathrm{Y}$ no es que uno (o una, en mi caso) ni mucho menos piense que solo en los países económicamente más poderosos haya académicos inteligentes, pero lo cierto es que tener los medios adecuados - una biblioteca en la que puedas pedir todo lo que acaba de publicarse y a la que lleguen las revistas electrónicas más importantes del momento, un despacho de calidad, clases bien equipadas, un internet que funcione con la rapidez necesaria - ayuda a tener la posibilidad de generar conocimiento de excelencia. Claro, que en el Viejo Mundo también hay diferencias, como las que yo he notado en el paso de dos de las mejores universidades del país, trasladándome de la Universidad de Durham a la Universidad de Barcelona. En todo caso, la falta de medios se puede suplir con tenacidad y con trabajo, aunque solo algunos lo suficientemente entusiastas como el prof. Funari lo logran.

Compruebo en su curriculum vitae que acabamos la tesis doctoral en el mismo año, 1990, aunque a él no le debían haber impuesto un límite de años para redactarla y por tanto algunos años más que yo tiene. Nuestros devenires han sido diferentes, puesto que él no se encontró con una universidad como era la española a principios de los noventa en la que no había ningún puesto de trabajo. Esto le permitió acceder a la carrera universitaria directamente en una universidad de reconocido prestigio, la UNICAMP. De libre-docente en Historia en 1996 pasaría a Profesor titular en 2004. Desde esta posición ha conseguido formar a un gran número de estudiantes a todos los niveles y de su valía hablan el que muchos de ellos hayan ahora encontrado puestos en la universidad no solo en Brasil en un número importante de centros, sino también en otros países como Colombia, Francia y Gran Bretaña, y sean mensajeros así de su labor docente.

\footnotetext{
1 Profesora Doctora, Universitat de Barcelona, Barcelona, España: email: m.diazandreu@ub.edu
} 


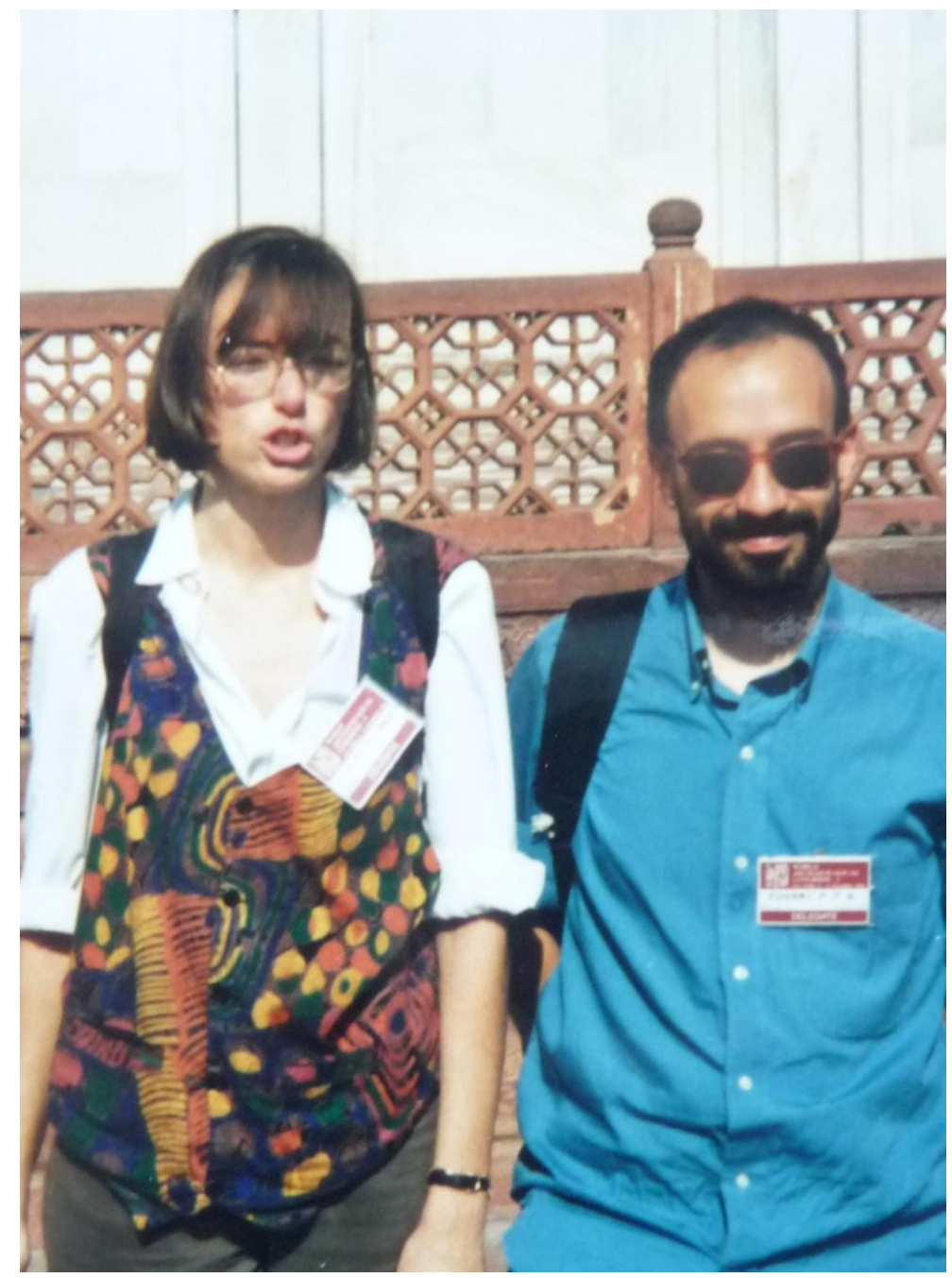

Fig. 1. Margarita Díaz-Andreu y Pedro Funari en la mezquita de Jama en Fetehpur Sikri, India, 1994.

Conocí al profesor Funari hace ya más de veinte años. Coincidimos en el año académico de 1992-93 en Inglaterra, él acogido por el University College London y yo por la Universidad de Southampton. Al estar conectados a través de Peter Ucko coincidimos en este y otros años en formar una serie de amistades internacionales en aquel momento unidas por el World Archaeological Congress (WAC). De ella destaco mi conexión con un gran número de académicos latinoamericanos que de ninguna otra manera pienso que habría yo conocido, además de otros europeos con los que ahora me relaciono más ahora a través de otro de los grandes congresos entonces surgidos: el de la Asociación Europea de Arqueólogos (la EAA, por sus siglas en inglés) con la que Pedro ha tenido menos que ver. Volviendo a WAC y a los noventa, Pedro y yo nos volvimos a encontrar en el congreso de 1994 en India (fig. 1). Yo tenía una presentación en una sesión sobre arqueología feminista y él supongo que presentaría su proyecto sobre la arqueología de los esclavos huidos en los Quilombos. De su participación en este congreso saldría luego publicado 
su artículo en el libro coordinado por él mismo y dos personas altamente vinculadas a WAC, Martin Hall, quien sería luego el presidente del WAC entre 1999 y 2003 y Siân Jones, esta última discípula de Peter Ucko (Funari et al., 1998). Los capítulos de este libro, como muchos de los otros editados por la serie One World Archaeology del WAC, ilustran bien la revolución que estaba teniendo lugar en arqueología y de la que ambos fuimos testigo y parte: no solo se incluían artículos de todo el mundo (combatiendo en gran manera - pero no totalmente - el insistente eurocentrismo que hasta entonces había caracterizado a los congresos internacionales) sino que se hablaba de política, imperialismo, género, etnicidad clases sociales, voces subalternas, historia oral, y de la arqueología de las minorías. Esto habría sido impensable antes de WAC y de esta revolución en el pensamiento arqueológico bebemos todavía hoy en día. En India al Prof. Funari se le nombraría el representante de América del Sur en el ejecutivo del WAC, cargo que mantuvo durante diez años hasta 2003, compaginando este con el de secretario de WAC entre 2002 y 2003.

En 1999 Pedro conseguiría financiación de la FAPESP (la Fundação de Amparo a Pesquisa do Estado de São Paulo) para que yo me trasladara a Brasil a impartir clases allí, lo que hice en el mismo São Paulo y sobre todo en la UNICAMP de Campinas y también brevemente en Joinville. He perdido casi toda memoria de los alumnos con los que entonces me encontré, lo que es una verdadera pena, porque me gustaría poderlos citar. En todo caso, de mi visita a Campinas de 1999 saldría publicado el artículo sobre "Nacionalismo y Arqueología: del Viejo al Nuevo Mundo" (Díaz-Andreu, 1999). Otra de las consecuencias de mi estancia de 1999 fue que en el curso 2003-2004 uno de los alumnos del Prof. Funari, Fabio Adriano Hering, vendría a la universidad de Durham, donde yo entonces trabajaba, a realizar una estancia. A Fabio sé que sí que lo había conocido durante mi estancia en Campinas en 1999, momento en el que él estaba terminando su maestría y había escrito, de alguna manera influido por lo que su maestro y yo misma le habíamos recomendado, un artículo sobre la influencia del nacionalismo en las construcciones discursivas de la Grecia Antigua (Hering, 2000, 2001, 2003). Durante su estancia, todavía estudiando a Herodoto y Tucídides, intentaba él ver la traducción que habían hecho los clasicistas británicos de estos autores y la influencia del nacionalismo y del racismo en sus puntos de vista. Tras su paso por Durham Fabio lograría terminar su tesis en 2006 y es actualmente profesor en la Universidade Federal de Ouro Preto. Fabio podría no haber sido el único alumno en venir a Durham bajo mi tutela. Vinieron otros, Andrés Zarankin y Ximena Senatore, pero por afinidad temática estaban tutorizados por mi colega Richard Hingley. Unos años más tarde nos 
planteamos el que viniera a Durham Andrés Alarcón pero finalmente aquello no cuajó. También me presentó a Gabriela Rodrigues quien participó en una sesión de la EAA de La Haya (Países Bajos) en 2010 y quien creo que ahora está en Heidelberg.

Pese a que él sea fundamentalmente un historiador de antigua y yo una prehistoriadora, nuestros intereses comunes han hecho que nuestras vidas académicas se hayan entrecruzado no solo en congresos sino también en publicaciones. Ya he comentado mi contribución a su libro de 1998 (Funari et al., 1998) y el artículo resultado de mi estancia en Brasil en 1999 (Díaz-Andreu, 1999). Luego hemos coincidido en libros editados sobre temas que nos interesan a los dos: historia de la arqueología, yo hablando de arqueólogas (Díaz-Andreu y Sørensen, 2008) y él de Brasil (Funari, 2008) y también Pedro tuvo la gentileza de sugerir mi nombre en un libro en el que se incluían relatos autobiográficos de mujeres arqueólogas que editaba él con la arqueóloga cubana Lourdes Domínguez y su alumna antes mencionada Gabriella Rodrigues (DíazAndreu, 2009). Por mi parte, yo le invité en el año 2009 a participar en una sesión en el congreso TAG (Theoretical Archaeology Congress) que realizamos con financiación de Santander Universidades analizando la relación entre turismo y arqueología (Funari et al., 2013).

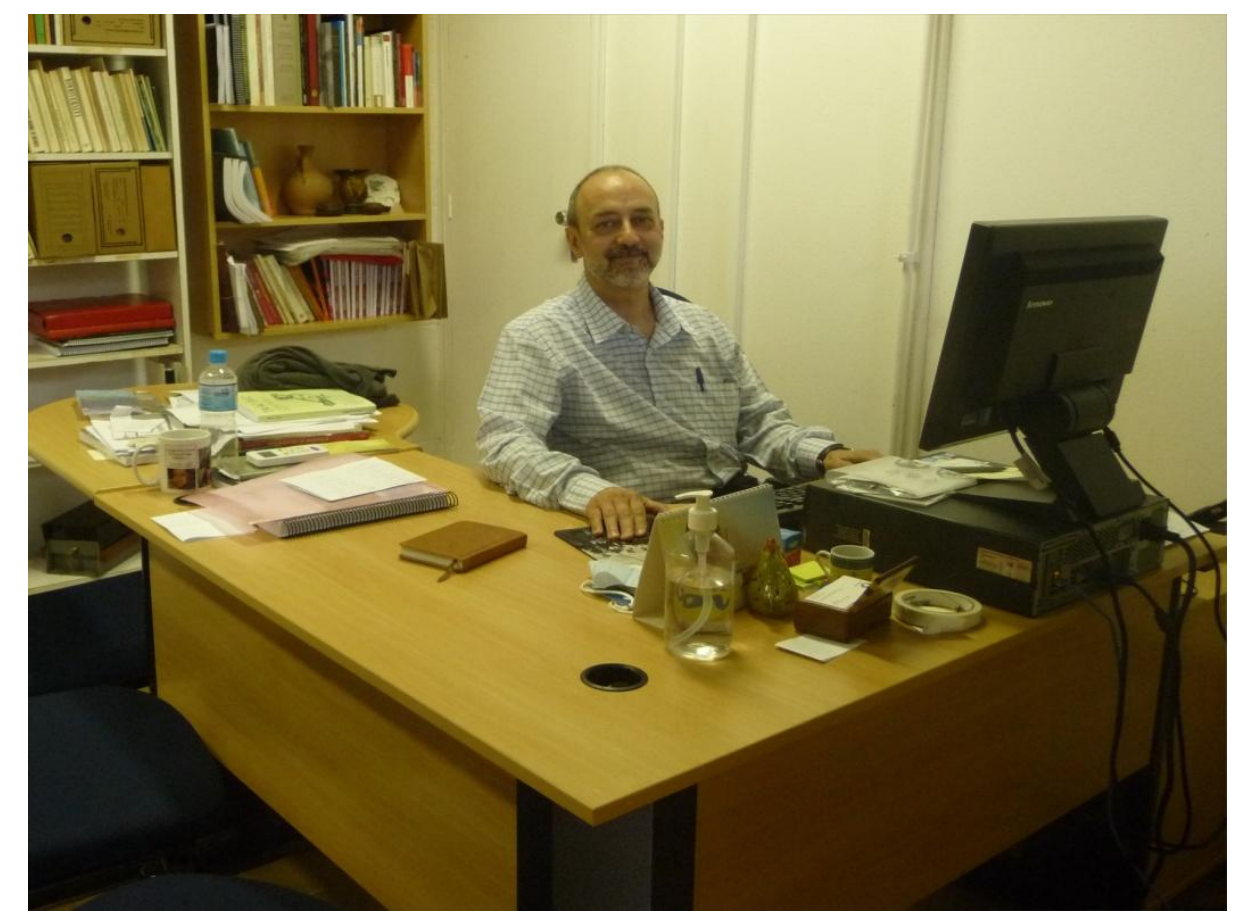

Fig. 2. Pedro Funari en su despacho de UNICAMP. 4 de noviembre de 2013 


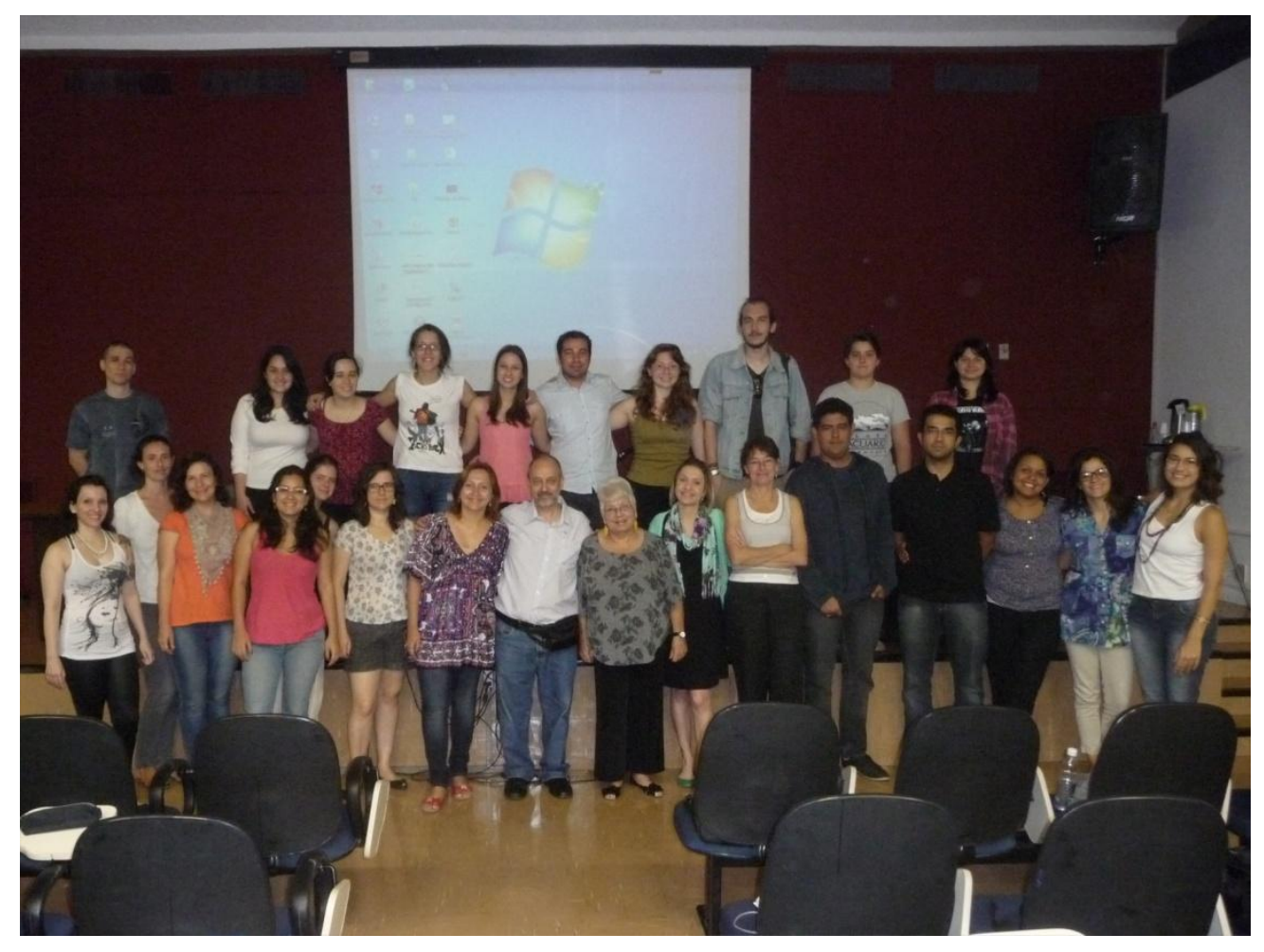

Fig. 3. Alumnos del mini-curso "Arqueología: cuestiones actuales" que impartí en la UNICAMP en noviembre de 2013

Volví a Brasil en 2013, momento en el que ya me encontraba en mi nueva etapa académica, basada en la Universidad de Barcelona como Profesora de Investigación ICREA financiada por el Instituto Catalán de Investigación y Estudios Avanzados. Invitada gracias a un Auxilio de Professor Visitante de la FAPESP organizado por Pedro Funari (figura 2) y por Aline V. Carvalho, tuve contacto con sus estudiantes Jocyane R. Baretta, Thiago do Amaral Biazotto, Natália Ferreira de Campos, Pedro Maguire Fermín, Victor Henrique S. Menezes, Rita Juliana Poloni, Tami Coelho Ocar, Rafael Augusto Nakayama Rufino, Isabela Soraia Backx Sanabria y Felipe N. Silva y con ellos debatí sobre sus proyectos de investigación, explicándome ellos lo que estaban haciendo y opinando yo sobre las posibilidades y orientaciones que veía en sus investigaciones. Además de los citados acudieron como oyentes en curso sobre "Arqueología: cuestiones actuales" otros alumnos como Taïs Pagoto Bélo, Marilin Calo, Cristina Fachini, Clarita Ferro, Isabela Barbosa Frederico, Camila Cassis Freitas, Mickaela Schwab Muñíz, Franciely Oliveira, Marcela Piccoli Pavao, Gabriela Pratavieira de Oliveira, Francisco de Souza Santos y Nara Witzler (figura 3). En este curso hablé sobre "Nacionalismo, colonialismo e imperialismo", "Los abusos de la arqueología" y "15 años de arqueología moderna y posmoderna". También tuve contactos con otros estudiantes como Patrícia Maurizzio, 
Cristina y quizá otros de cuyo nombre que ahora no logro acordarme, incluyendo aquéllos que me enseñaron el Laboratorio de Arqueología (figura 4). En todo momento estuve acompañada por la arqueóloga cubana ya citada más arriba, invitada también en esos meses en la Universidad de Campinas, Lourdes Domínguez (figura 5). Durante esta estancia de 2013 también impartí conferencias en centros donde ahora trabajaban antiguos alumnos del Profesor Funari, en la Universidade de São Paulo invitada por Fabiana Manzato y en la Universidade de Pelotas donde me acogió Lúcio Ferreira. En esta última ciudad además se me dio la oportunidad de participar en el $7^{\circ}$ Simposium / Convenção do Patrimônio Imaterial que allí organizaba la profesora Maria Leticia Mazzucchi Ferreira. He de apuntar además que desde el 2013 he tenido reuniones en Barcelona con dos de sus alumnos (Rafael Augusto Nakayama y Felipe N. Silva) que han venido a hacer estancias de investigación en Barcelona no bajo mi amparo, sino del Profesor José Remesal, con quien el Profesor Funari, como historiador de antigua, tiene una relación muy estrecha desde hace por lo menos dos décadas.

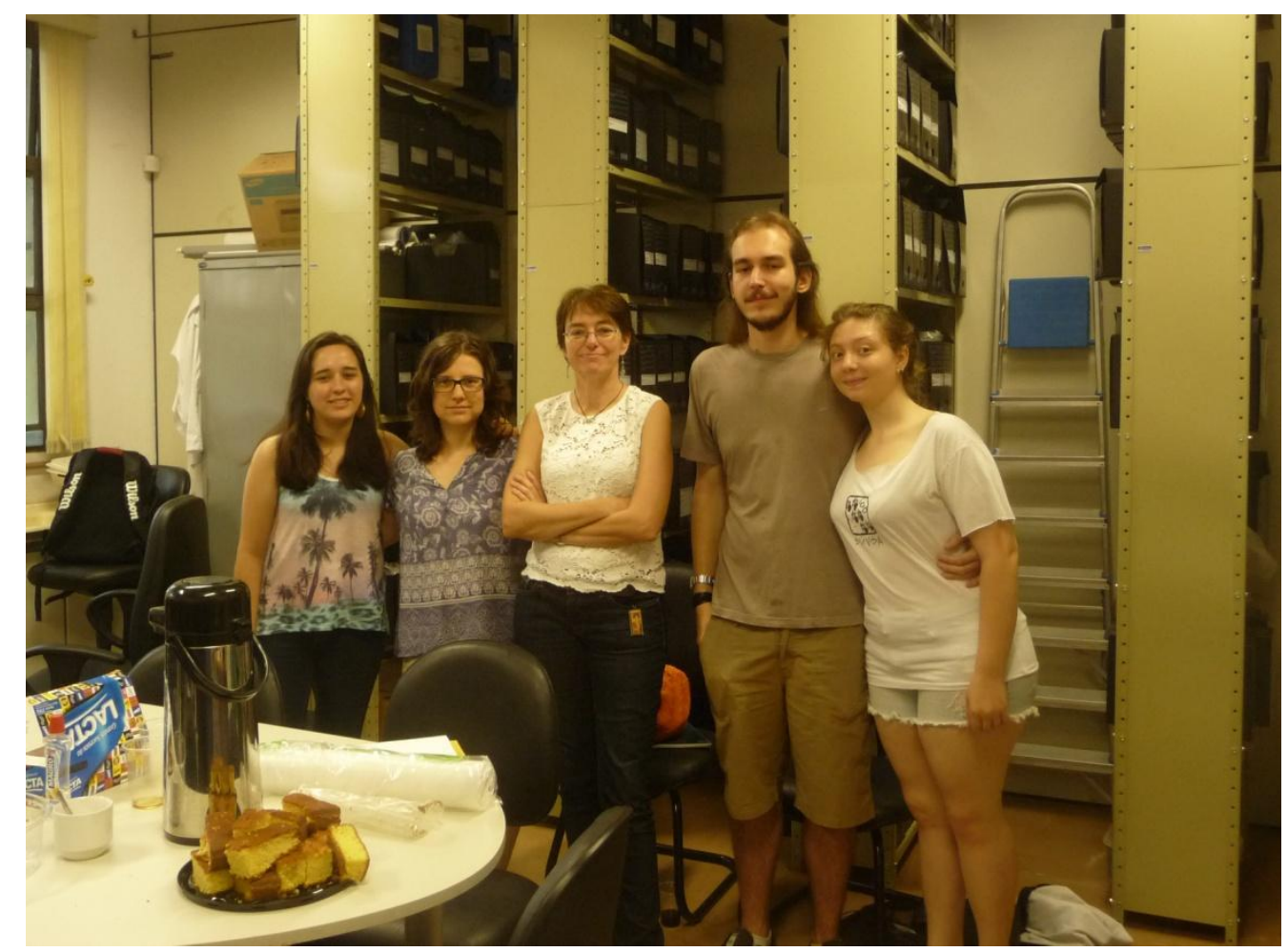

Fig. 4. Visita al Laboratorio de Arqueología de UNICAMP. Noviembre de 2013.

En estos últimos años he investigado sobre cómo el conocimiento se traslada entre unas partes y otras del mundo y cómo la geografía es un condicionante, aunque no un límite, en este movimiento. Muchas de las ideas expresadas en un libro que ahora ya tiene unos años (Díaz-Andreu, 
2012) se podrían aplicar al estudio del quehacer y de la obra del profesor Funari. En él vemos cómo las relaciones internacionales entre arqueólogos influyen en el movimiento de ideas: su estancia en Southampton (1988), sus estudios en Illinois (1991-92) y en Londres, en UCL (1992-93), le abrieron puertas y sobre todo la de un congreso internacional, el World Archaeological Congress, que le permitió ser parte de una plataforma de diálogo intercontinental de dimensiones que no creo que él se hubiera podido imaginar anteriormente. Así vemos cómo se expandieron sus horizontes que pasaron de ser exclusivamente la historia antigua. No es que todo fuera nuevo para él puesto que su decisión de trasladarse al extranjero parecía alentada por una curiosidad que ya le llevaba más allá y de ello son testigo sus tempranos trabajos sobre democracia (Funari, 1987), teoría (Funari, 1990b) o educación (Funari, 1990a). A su primera contribución al World Archaeological Bulletin de 1989 (Funari, 1989) le siguen otras (Funari, 1991a, 1996, 2000, 2001) que tienen el regusto de la organización, con su obsesión, al menos en aquel entonces, en la política. Por influencia de las nuevas ideas que está recibiendo se interesa por la arqueología de las Américas y especialmente la de los desfavorecidos, incluyendo la población esclava negra, de la que vemos sus primeros trabajos a principios de los años noventa en un proyecto en el que colabora fundamentalmente con Charles E.Orser (Funari, 1991b, 1995; Funari y Orser, 1992) y sobre el que más tarde y hasta hoy publicará sobre otros temas asociados (Dominguez y Funari, 2005; Funari y Domínguez, 2006; Menezes Ferreira et al., 2016).

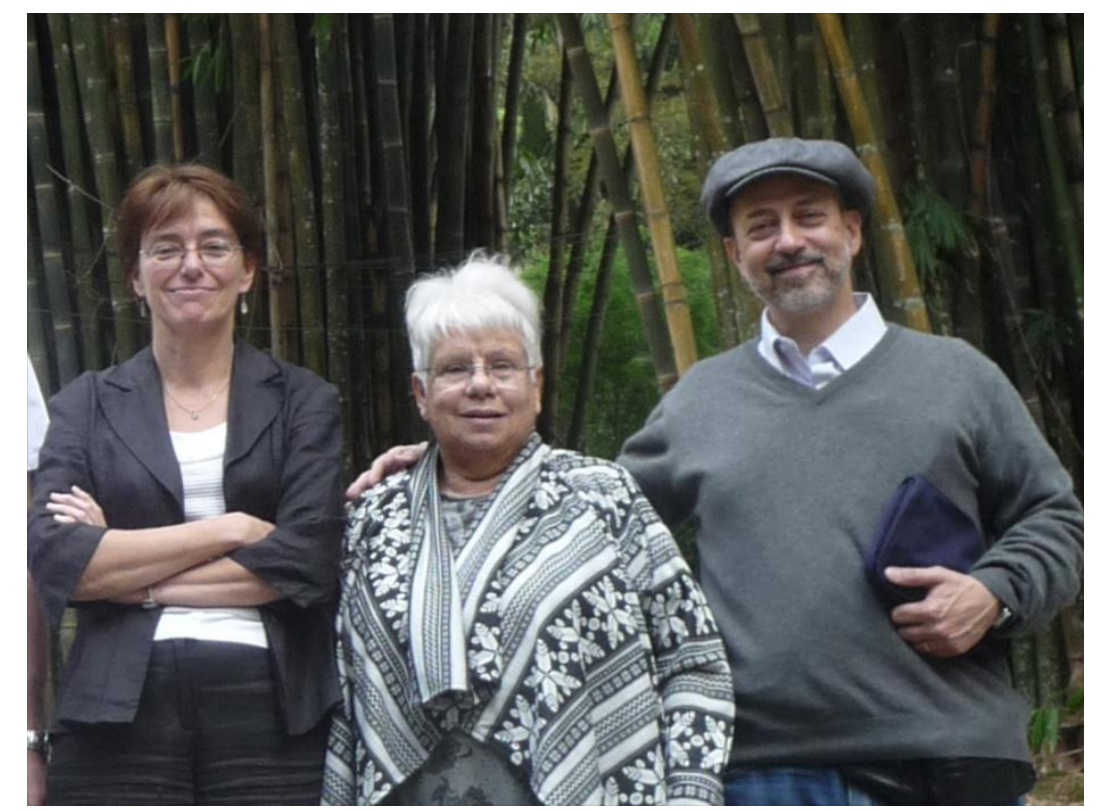

Fig. 5. Margarita Díaz-Andreu, Lourdes Domínguez y Pedro Funari en el exterior del comedor universitario de UNICAMP. 1 de noviembre de 2013. 
Importante en sus publicaciones es observar cómo lo que él ha absorbido fuera de Brasil se lo transmite a sus alumnos, con los que frecuentemente publica y así vemos cómo puede disertar sobre temas muy diversos de los que frecuentemente se convierte en pionero en Brasil: arqueología urbana (Dominguez y Funari, 2002; Funari, 2015; Funari y Zarankin, 2003), arqueología pública (Funari y Robrahn-González, 2007), arqueología histórica (Funari, 2010), identidad (Funari et al., 2005), colonialismo (Funari y Senatore, 2015), arqueología pública (Funari et al., 2015a) y participativa (Funari y Garraffoni, 2016), museos (Funari et al., 2015b), educación (Funari, 2004), turismo arqueológico (Funari et al., 2015c; Manzato y Funari, 2011), derechos humanos (Soares y Funari, 2014), inclusión social (Funari y Tega, 2014) y otro elenco de temas que vienen a complementar su investigación continuada en Historia antigua y el uso que de esta se hace, en solitario o con alumnos (por ejemplo Garraffoni y Funari 2012). Volviendo a la geografía del conocimiento me parece importante apuntar que esta gran cantidad de saberes sería impensable en los centros del poder mundial en la ciencia, los países donde se produce una inversión estatal mucho más cuantiosa que en Brasil, y donde entonces la especialización de cada profesional es más limitada. Las condiciones en las que ha trabajado el Profesor Pedro Funari, muy contrarias a la abundancia económica del país donde vive, han hecho que él se concentren muchos saberes y que sus alumnos hayan tenido la suerte de acceder a través de él a un gran abanico de conocimiento que en otros lugares habrían obtenido solo en sus contactos con un mayor número de académicos. De ello se deduce que lo que no permite la economía lo produce el tesón y el trabajo y Pedro ha sido durante años un ejemplo claro del sabio inquieto que no se guarda el saber para sí, sino que lo transmite a los que están a su alrededor. Esta transmisión es la que yo he experimentado yo también en mis estancias en Brasil: en las que yo he servido de cauce de conocimientos que proporcionaba a los alumnos que allí me escuchaban y a todos aquellos que luego me visitaban en donde estuviera, en Durham o en Barcelona, pero a la vez yo también he también me he instruido de todos ellos. Acabo, por tanto, como he empezado, expresando mi admiración al maestro Funari, a su capacidad de transmitir conocimiento no solo a aquellos que le hemos conocido sino sobre todo a sus alumnos y alumnas, proporcionándoles una formación y nivel académico que en pocos otros lugares podrían adquirir.

\section{Bibliografía}

Díaz-Andreu, M. Nacionalismo y Arqueología: del Viejo al Nuevo Mundo In: Funari, P.P.A., Neves, E.G., Podgorny, I. (Eds.), Anais da I 
Reunião de Teoria Arqueológica na América do Sul, São Paulo. Museu de Arqueologia e Etnologia da Universidade de São Paulo, Revista do Museu de Arqueologia e Etnologia, Suplemento 3, São Paulo, 1999, pp. 161-180.

DÍAZ-ANDREU, M. Reflexión de una arqueóloga española en el Reino Unido, In: Domínguez, L.S., Funari, P.P.A., Carvalho, A., Rodrigues, G. (Eds.), Desafios da Arqueologia: Depoimentos. Editora Habilis, Erechim, 2009. pp. 157-161.

DÍAZ-ANDREU, M. Archaeological encounters. Building networks of Spanish and British archaeologists in the 20th century. Cambridge Scholars, Newcastle, 2012.

DÍAZ-ANDREU, M., Sørensen, M.L. Excavating Women: Towards an Engendered History of Archaeology, In: Murray, T., Evans, C. (Eds.), Histories of archaeology: a reader in the history of archaeology. Oxford University Press, Oxford, 2008. pp. 279-311.

DOMINGUEZ, L., FUNARI, P.P.A. La Arqueología Urbana en América Latina: el caso de Habana Vieja, ciudad arqueológica. Estudos IberoAmericanos 28, 2002, 113-124.

DOMINGUEZ, L., FUNARI, P.P.A. La Arqueología de Brasil y Cuba, en tiempos de la esclavitud. Noticias de la Universidad de Tula, Historia y Cultura, Tula, Rússia 3, 2005, 79-100.

FUNARI, P.P.A. Antiguidade, Proposta Curricular e Formacao de Uma Cidadania Democratica. Boletim do CPA (UNICAMP) 7, 1987, 261-262.

FUNARI, P.P.A. Brazilian Archaeology And World Archaeology: Some Remarks. World Archaeological Bulletin 3, 1989, 60-68.

FUNARI, P.P.A. Education Through Archaeology: A Bumpy But Exciting Road. Archaeology and Education 1, 1990a ,9-11.

FUNARI, P.P.A. Reflexões Sobre A Mais Recente Teoria Arqueologica. Revista de Pre-Historia 7, 1990b, 203-209.

FUNARI, P.P.A. Archaelogy In Brazil: Politics And Scholarship At A Crossroads. World Archaeological Bulletin 5, 1991a., 122-132.

FUNARI, P.P.A. A Arqueologia e A Cultura Africana nas Américas. Revista de História Regional 17, 1991b, 61-71. 
FUNARI, P.P.A. The Archaeology Of Palmares And Its Contribution To The Understanding Of The History Of African-American Culture. Historical archaeology in Latin America 7, 1995, 1-41.

FUNARI, P.P.A. Historical Archaeology In Brazil, Uruguay And Argentina. World Archaeological Bulletin 7, 1996, 51-62.

FUNARI, P.P.A. Report on the publication of the Proceedings of the First International Meeting on Archaeological Theory in South America. World Archaeological Bulletin 12, 2000, 96-99.

FUNARI, P.P.A. Review of "Archaeology under fire, nationalism, politics and heritage in the Eastern Mediterranean and Middle East, ed. L. Meskell. World Archaeological Bulletin 13, 2001, 82-88.

FUNARI, P.P.A. Patrimônio: uma educação para a cidadania. Vitrivius 89, 2004.

FUNARI, P.P.A. A History of Archaeology in Brazil, In: Murray, T., Evans, C. (Eds.), Histories of archaeology: a reader in the history of archaeology. Oxford University Press, Oxford, 2008, pp. 328-345.

FUNARI, P.P.A. To whom belongs Brazilian archaeological remains: the role of public archaeology, In: Funari, P.P.A., Oliveira, N., Zarankin, A., Senatore, X., Dominguez, L. (Eds.), Contemporary Issues in Historical Archaeology. Archaeopress, Oxford, 2010, pp. 9-14.

FUNARI, P.P.A. História Comparada en Iberoamérica: las ciudades españolas y portuguesas en el Nuevo Mundo. Revista de História Comparada (UFRJ) 9, 2015, 69-87.

FUNARI, P.P.A., Campos, J.B., Rodrigues, M., Arqueologia Pública e Patrimônio: questões atuais. Ediunesc, Criciúma, 2015a.

FUNARI, P.P.A., DOMÍNGUEZ, L. El método arqueológico en el estudio de la esclavitud en Cuba y Brasil. Boletín del Gabinete de Arqueología 5, 2006, 52-65.

FUNARI, P.P.A., GARRAFFONI, R.S. Arqueología participativa y empoderamiento comunitario en Brasil. Complutum [Dossier: Castillo, A. (ed.) 2016. Interpreting the Past through Participatory Approaches: Ideals and Challenges in Archaeological Practice] 27, 2016, 281-294.

FUNARI, P.P.A., Hall, M., JONES, S. Historical Archaeology. Back from the Edge. Routledge, London, 1998. 
FUNARI, P.P.A., MANZATO, F., ALFONSO, L.P. Tourism and Archaeology in Brazil: Postmodern Epistemology in Two Case Studies International Journal of Historical Archaeology [special issue: DÍAZANDREU, M. y Villalobos Acosta, C. (eds.). The ethics of archaeological tourism in Latin America] 17, 2013, 261-274.

FUNARI, P.P.A., MANZATO, F., ALFONSO, L.P. El turismo y la arqueología en el Brasil: una mirada posmoderna, In: Herrera Wassilowsky, A. (Ed.), Arqueología y desarrollo en América del Sur. De la práctica a la teoría. Universidad de los Andes, Instituto de Estudios Peruanos, 2015c , p. PAGES.

FUNARI, P.P.A., Orser, C.E. Pesquisa arqueológica inicial em Palmares. Estudos Ibero-Americanos, Porto Alegre 18, 1992. 53-69.

FUNARI, P.P.A., Orser, C.E., SCHIAVETTO, S.N.O. Identidades, discurso e poder: Estudos da arqueologia contemporânea. Sao Paulo, FAPESP, Annablume, 2005.

FUNARI, P.P.A., ROBRAHN-GONZÁLEZ, E.M. Editorial: Arqueologia Pública na América Latina. Arqueologia Pública (UNICAMP) 2, 3-4, 2007.

FUNARI, P.P.A., SENATORE, M.X. Archaeology of culture contact and colonialism in Spanish and Portuguese America. Springer, New York, 2015.

FUNARI, P.P.A., TEGA, G. Arqueologia, do imperialismo à inclusão social. Expressa Extensão (UFPel) 19, 2014, 17-27.

FUNARI, P.P.A., VASCONCELLOS, C.M., CARVALHO, A.V. Museus e Identidades na América Latina. Annablume, São Paulo, 2015b.

FUNARI, P.P.A., ZARANKIN, A. Social archaeology of housing from a Latin American perspective: A case study. Journal of Social Archaeology 3, 2003, 23-45.

GARRAFFONI, R.S., FUNARI, P.P.A. The uses of Roman heritage in Brazil. Heritage and Society 5, 2012, 53-76.

HERING, F.A. O papel do nacionalismo nas construções discursivas acerca da Grécia Antiga: apontamentos iniciais. Boletim do CPA 10, 2000, 57-68.

HERING, F.A. M. I. Rostovtzeff e uma Arqueologia Nacionalista do Sul da Rússia - ou dos usos ideológicos da Narrativa de Heródoto. LPH Revista de História 11, 2001, 17-32. 
HERING, F.A. O exílio de Heródoto: do juízio de Tucídides à sua apropriação moderna, In: Lopes, M.A. (Ed.), Grandes Nomes da História Intelectual. Editora Contexto, São Paulo, 2003.

MANZATO, F., FUNARI, P.P.A. Turismo e a aproximação das culturas evidenciadas nas práticas de compartilhamento do patrimônio arqueológico. Turismo e Sociedade 4, 2011, 186-199.

MENEZES FERREIRA, L., FUNARI, P.P.A., ALVES, A.G. La Arquología de los Quilombos en Brasil. Revista Euroamericana de Antropologia Salamanca 2016, 68-80. 